\title{
Téoros
}

Revue de recherche en tourisme

\section{Accessibilité forestière accrue} Panacée ou boîte de Pandore ?

\section{Daniel Kneeshaw et Sylvie Gauthier}

Volume 25, numéro 3, automne 2006

Tourisme et forêt, visions d'une gestion intégrée

URI : https://id.erudit.org/iderudit/1071017ar

DOI : https://doi.org/10.7202/1071017ar

Aller au sommaire du numéro

Éditeur(s)

Université du Québec à Montréal

ISSN

0712-8657 (imprimé)

1923-2705 (numérique)

Découvrir la revue

Citer cet article

Kneeshaw, D. \& Gauthier, S. (2006). Accessibilité forestière accrue : panacée ou boîte de Pandore ? Téoros, 25(3), 36-40. https://doi.org/10.7202/1071017ar d'utilisation que vous pouvez consulter en ligne.

https://apropos.erudit.org/fr/usagers/politique-dutilisation/ 


\section{Accessibilité forestière accrue Panacée ou boîte de Pandore?'}

\section{Daniel Kneeshaw et Sylvie Gauthier}

Dans les années 1970, la forêt québécoise était peu accessible à la population. À cette époque, le réseau routier était peu développé, sauf dans le sud du Québec. II fallait donc disposer de bons moyens financiers pour se faire transporter en hélicoptère ou en hydravion vers les clubs de chasse ou encore être prêt à consacrer beaucoup de temps et d'énergie pour visiter les zones forestières (dans le cas de certains adeptes de plein air à la recherche de contrées sauvages par exemple). Sinon, il fallait vivre des ressources de la forêt (autochtones, forestiers, etc.). Certaines régions, colonisées il y a longtemps, étaient relativement accessibles, mais la grande forêt québécoise, en particulier la forêt boréale, est demeurée une chasse gardée pour les concessions forestières et les clubs privés. Avant l'avènement du tourisme de nature, on a voulu assurer l'accès au territoire à toute la population en abolissant les concessions. Le gouvernement a ainsi favorisé une démocratisation de la forêt en participant à la construction d'un réseau routier très développé.

Or, quelques décennies plus tard, la situation a passablement changé. Aujourd'hui, il ne reste que peu d'endroits non accessibles par des chemins et les routes sont construites à un rythme trois fois plus élevé que dans les années 1970 (voir Bourgeois et al., 2006 pour d'autres statistiques). L'accessibilité au territoire a donc considérablement augmenté et la mentalité voulant qu'on ouvre de plus en plus le territoire est bien instaurée. Les arguments sont assez simples : les compagnies forestières veulent accéder aux forêts mûres et non coupées afin d'approvisionner leurs usines et le gouvernement veut faire rouler l'éco-

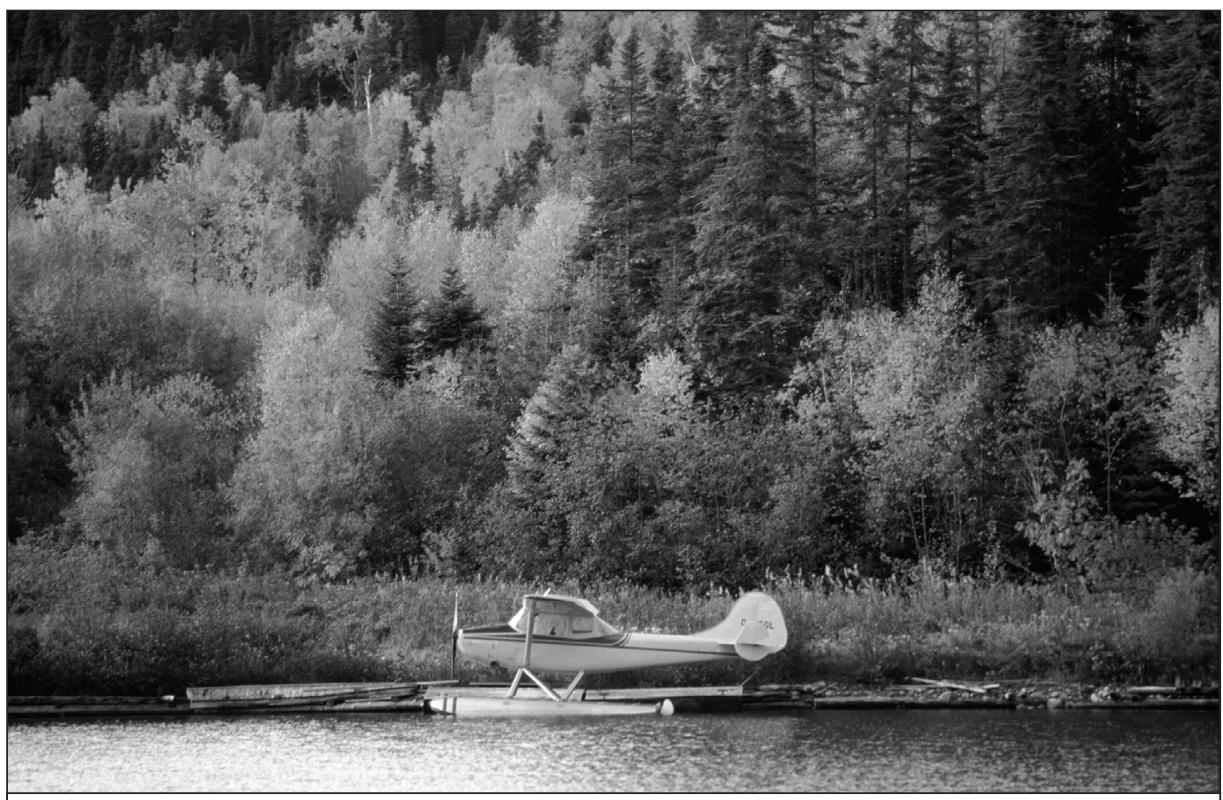

Hydravion sur un lac de Forestville dans la région touristique Manicouagan (Québec). Photo: Michel Julien/Tourisme Québec

nomie des régions. D'autres justifications, telle une meilleure protection des forêts contre les incendies de forêt, sont parfois apportées. Même si ces arguments sont valables, la situation changeante en ce qui concerne l'aménagement forestier et les désirs de la société suggère qu'il est temps de réévaluer et de mieux planifier l'accessibilité au territoire. On sous-entend généralement qu'un réseau routier très développé est bénéfique pour la société et l'économie sans porter un regard critique sur les aspects positifs et négatifs de ce développement. En ce moment, au Québec, le développement se poursuit sans aucune planification, alors qu'aux États-Unis et dans plusieurs provinces à l'ouest du Québec, on signale les dangers qu'une telle approche pourrait avoir sur la biodiversité ainsi que d'un point de vue socioéconomique (Bourgeois et al., 2006).
Tourisme et accès routier au nord

Une des régions qui risque le plus d'être affectée par une expansion du réseau routier est la forêt boréale. Dans la conscience (ou l'inconscient) collective, cette forêt nordique est tellement vaste qu'il est à peu près impossible de l'altérer en entier par des actions anthropiques. Les enjeux ne sont toutefois pas les mêmes dans le sud de la province, plus densément peuplé. Des facteurs comme la facilité d'accès, la proximité des marchés, la concentration de la demande à certaines périodes (été et fins de semaine) et la popularité de certaines activités peuvent créer des pressions importantes sur les écosystèmes du sud. Par contre, au nord, l'achalandage est plus restreint, la clientèle différente (surtout des Européens, des Américains et d'autres Canadiens qui dépensent d'importantes sommes d'argent) (Tourisme Québec, 2003 : 68) et les vastes 
espaces sont encore relativement naturels et calmes. Par ailleurs, la forêt boréale est principalement un territoire public où les usages multiples et le manque d'aires conservées constituent des enjeux importants (Couture, 2002 : 43).

\section{Image du Québec: tourisme vs foresterie}

Le ministère du Tourisme au Québec vend, avec succès, l'image de vastes territoires non exploités et des visiteurs dépensent des milliers de dollars chacun pour voir ces territoires. En fait, Tourisme Québec (2003 : 26) mentionne qu'un des principaux facteurs qui déterminent la capacité d'une destination à attirer des visiteurs est la qualité de l'environnement naturel et des paysages. L'industrie touristique constitue un moteur important de développement économique au Québec : en 2004, ce sont 133500 emplois directs et des retombées de plus de 9 millions de dollars (Tourisme Québec, 2004 : 1). Par ailleurs, une étude récente conclut que l'écotourisme et le tourisme de nature comptent pour $10 \%$ des dépenses touristiques (Mathieu, 2004). En forêt, les visiteurs proviennent de différents groupes : les observateurs de la nature (ornithologues amateurs, par exemple) et ceux qui veulent profiter des éléments physiques (randonnée, escalade, canotage, etc.).

Les chasseurs et les pêcheurs ne sont pas inclus dans ce rapport, mais ils s'ajoutent aux utilisateurs de la forêt et contribuent beaucoup à l'économie régionale. Un rapport sur l'utilisation des territoires fauniques souligne qu'en 2000 plus de 7600 emplois, plus de 103 millions de dollars en salaires et plus de 181 millions de dollars en PIB (produit intérieur brut) ont été générés par les organismes dédiés à la faune (ZEC², parcs, réserves fauniques et pourvoiries) (Société de la faune et des parcs du Québec, 2003 : 3). Une grande partie de cet argent et de ces emplois sont générés dans les régions éloignées où la foresterie est aussi importante. Certains utilisateurs préfèrent un accès facile par le réseau routier, mais d'autres préfèrent les milieux sans route où ils ont l'impression d'être dans une grande forêt naturelle qui n'a pas été affectée par les activités humaines industrielles. L'accès restreint au territoire a en fait été identifié comme un élément important pour préserver l'habitat de la faune, mais aussi pour la quiétude des lieux (Fédération des pourvoiries du Québec, 2004 : 4).

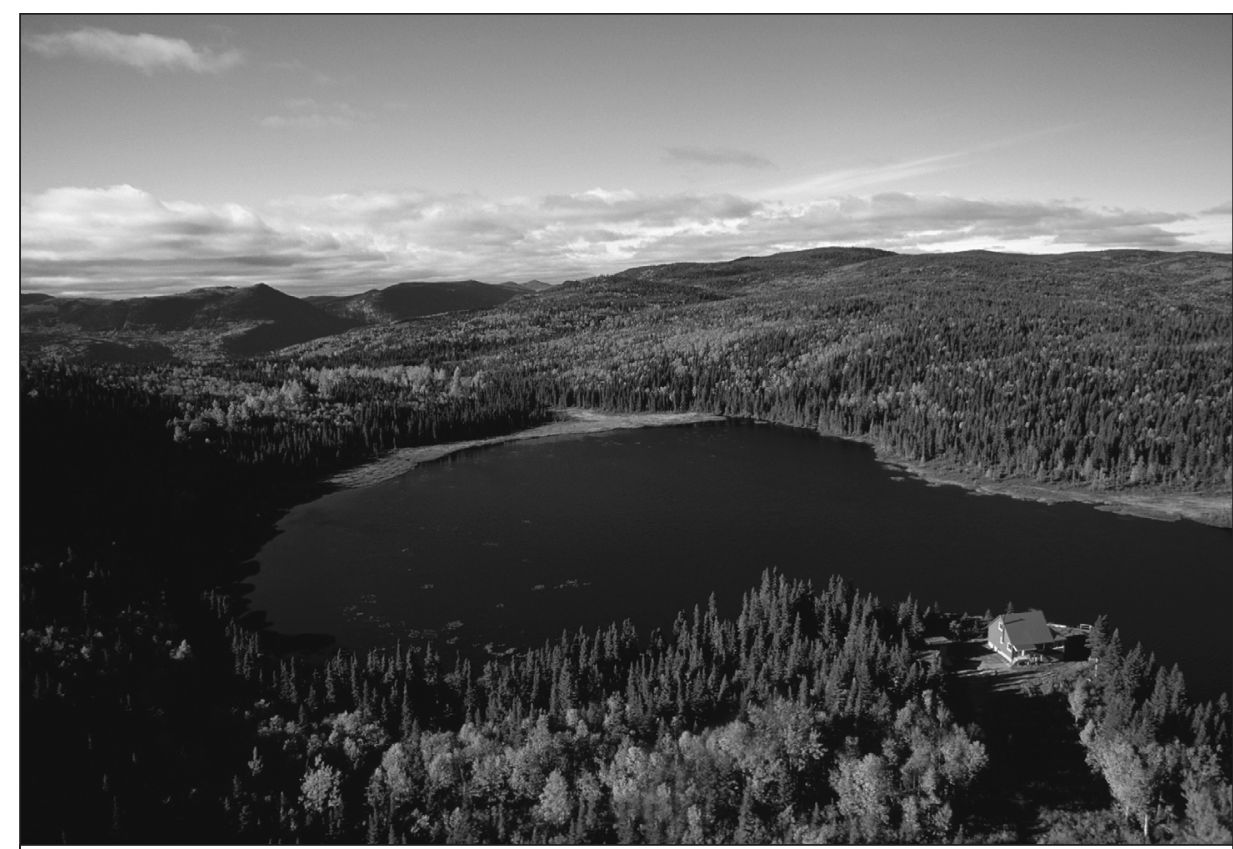

Paysage aérien de la région de la Jacques-Cartier (Québec).

Photo: Yann Arthus-Bertrand/Tourisme Québec

D'autres documents font état des conséquences potentiellement néfastes du réseau routier pour certaines espèces fauniques (Bourgeois et al., 2006 ; Forman, 2000 : 31 ; Trombulak et Frissell, $2000: 18$ ). Des exemples d'effets négatifs comprennent: la fragmentation du territoire, l'introduction d'espèces exotiques, l'altération du comportement animal (par exemple le bruit causé par les véhicules), les effets directs et indirects de l'augmentation de la présence humaine (braconnage, collisions, dérangement des activités des animaux, allumage d'incendies), les pertes d'habitat, etc. Évidemment, il faut bien soupeser les effets des routes qui peuvent être, d'une part, positifs en termes d'accès et, d'autre part, négatifs pour la faune (collisions, braconnage, dérangement de comportement, etc.) que les gens viennent justement observer.

Des données suggèrent aussi que l'utilisation du territoire à des fins d'observation ou sportives sans prélèvement est à la hausse. Marcoux (1993: 18) rapporte qu'au début des années 1990 moins de $10 \%$ des clients qui fréquentaient les pourvoiries y étaient pour l'observation de la nature. Depuis ce temps, cette proportion aurait beaucoup augmenté et atteindrait les $25 \%$ environ (Fédération des pourvoiries du Québec, 2004: 1).
Les valeurs et l'économie associées à la faune et au tourisme de nature sont en hausse. La cohabitation des différents utilisateurs doit maintenant être facilitée par les entreprises qui font de l'aménagement forestier. Ainsi, pour un territoire donné, les ententes sont généralement conclues localement. Par contre, à l'échelle de la forêt boréale québécoise, c'est le gouvernement qui propose des lignes directrices. Par exemple, l'utilisation de la coupe mosaïque (Leduc et al. , 2002 : 20) qui occasionne des coupes plus petites, mais plus dispersées, et nécessite donc un réseau routier bien développé, a été imposée par le gestionnaire de la forêt. Même si cette forme d'aménagement est avantageuse pour certaines espèces fauniques, elle pourrait avoir des effets négatifs sur d'autres groupes (par exemple, les espèces qui tolèrent mal la fragmentation ou l'effet des routes). Souvent, ces directives sont présentées comme solution à un problème. Cependant, le manque de réflexion approfondie pourrait apporter de nouveaux problèmes (Commission Coulombe, 2004 : 50). De plus, il peut y avoir des lacunes importantes dans la planification à certaines échelles. Par exemple, la mise en place du réseau routier dans deux territoires d'aménagement est faite de manière indépendante (deux territoires forestiers ou un territoire forestier adjacent à une zone de conservation, par exemple), il n'y a presque pas de planification qui tienne compte des aménagements dans le territoire adjacent. 


\section{Concertation?}

On en vient donc à se demander s'il existe une stratégie concertée entre les différentes instances gouvernementales lorsqu'il s'agit d'accroître le tourisme de nature et la construction de routes tout en assurant une foresterie viable à long terme. Les ministères qui visent le développement des ressources comme la foresterie, les mines et les barrages hydroélectriques communiquent-ils avec les ministères qui veulent promouvoir les grands espaces naturels au Québec? Un des plus grands défis du développement du tourisme de nature est I'harmonisation des besoins pour les différents utilisateurs de la forêt (Couture, 2002 : 43). La qualité des expériences de tourisme de nature dépendrait des bonnes pratiques et d'une vision qui tienne compte de toutes les activités en milieu naturel. Y a-t-il incompatibilité entre les différents usages et existe-t-il des solutions gagnantes pour résoudre ces différences?

Afin d'assurer une planification qui tienne compte des diverses avenues de développement des régions forestières du Québec, il faut d'abord déterminer si une accessibilité accrue est souhaitable ou non. II est indéniable qu'un réseau routier développé comporte de nombreux avantages : des régions, qui autrement seraient isolées, sont ainsi relativement accessibles au grand public. Néanmoins, il semble que l'expérience dans son ensemble (nature, tranquillité, atmosphère paisible et calme, etc.) est ce qui détermine le succès d'une visite d'écotourisme (Lequin et Carrière, 2003 : 29).

Malheureusement, certaines pratiques actuelles d'aménagement obligent à faire de longs détours par avion pour éviter que les touristes ne voient les grandes coupes forestières et les nombreuses routes qui traversent la forêt. Le fait d'apercevoir en voiture les coupes derrière les minces lisières d'arbres laissés après la récolte, le manque de lisière tout court ou encore le bruit des véhicules sur les routes lors d'un séjour en forêt sont autant de facteurs qui affectent négativement une expérience en nature. Le marché de l'écotourisme est marqué par une compétition internationale féroce et l'élaboration de stratégies pour minimiser ces nuisances est impérative. Le ministère responsable du tourisme de nature a compris la nécessité de sensibiliser les entreprises et les organismes impliqués à adop- ter des pratiques qui contribueront à la conservation et à l'utilisation rationnelle des ressources naturelles du Québec (Tourisme Québec, 2003 : 26). II est grand temps que le dialogue entre les différents ministères et entre les entreprises et les parties prenantes soit établi.

\section{Stratégies pour atténuer l'effet des routes}

II serait donc utile, voire essentiel, de mieux planifier l'accès au territoire de manière à en tirer des bénéfices tout en minimisant les impacts négatifs. Nous suggérons une stratégie hiérarchique qui vise entre autres la conservation de territoires sans accès routier et la mitigation des effets négatifs sur les zones accessibles. Une stratégie de développement intégrant les besoins des différents utilisateurs reposerait d'abord sur un zonage du territoire (Commission Coulombe, 2004 : 80, Messier et Kneeshaw, 1999: 929), sur la renaturalisation des routes lorsque possible et sur la mitigation des impacts.

La première étape de la stratégie, le zonage, est une approche déjà préconisée et appliquée en partie. Cette approche reconnaitt que l'exploitation prioritaire par une seule industrie (d'une seule ressource) causerait des inconvénients aux autres utilisateurs du milieu naturel. Afin de respecter les lieux convoités pour leur caractère naturel et de combler les besoins de tous les usagers, certaines zones devraient être exemptes d'opérations forestières et d'accès routier. Une perte de récolte de matière ligneuse serait alors acceptée aux endroits désignés. Cette perte pourrait cependant être balancée par un aménagement plus intensif ailleurs (autour des usines par exemple) (Messier et al., 2003 : 61 ; Seymour et Hunter, 1992). Bien entendu, il n'y aura pas un véritable partage de la ressource si un seul type d'usage demeure prioritaire. Le zonage permettrait de prioriser différentes utilisations sur différentes parties du territoire, sans forcément exclure les autres types d'utilisations. Par exemple, à l'exception des aires de conservation intégrale, une partie du territoire peut être affectée à une utilisation forestière en priorité, avec le tourisme de nature au deuxième rang et d'autres utilisations au troisième rang. Un autre secteur pourrait, en contrepartie, être zoné pour l'écotourisme en priorité avec la foresterie au deuxième rang. Un tel zonage pourrait nécessiter des pratiques forestières plus douces et plus harmonieuses (pour maintenir un couvert continu) et un réseau routier planifié de façon à promouvoir des objectifs écotouristiques (densité de chemins moins élevée, plus de culs-de-sacs, décommissionnement des routes, etc.)

Certaines zones devraient aussi être désignées comme aires de conservation, sans accès routier. II y a un manque flagrant

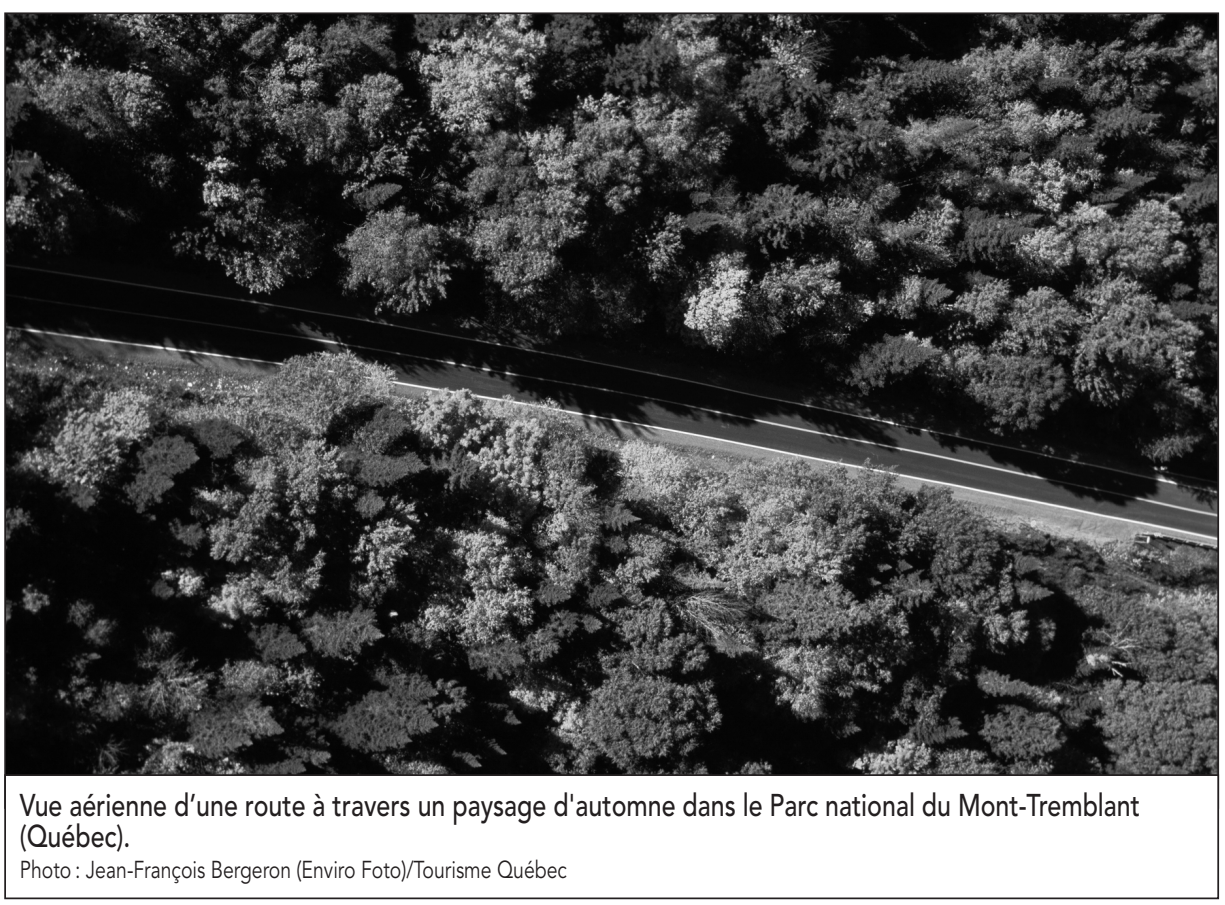


d'aires de conservation sur le territoire québécois, particulièrement dans la zone de forêt commerciale du nord. La commission Coulombe (2004 : 53) a constaté ce manque et souligne que le gouvernement se doit de faire un effort pour atteindre l'objectif mondial de $12 \%$ d'aires de conservation dans la zone boréale au lieu de l'objectif actuel de $8 \%$. Les aires de conservation devront comprendre des zones représentatives plutôt que seulement des lieux ayant des caractéristiques uniques (Bonneau et Kneeshaw, 2004 : 30). II faut mentionner qu'en plus des aires de conservation intégrale, le Québec comporte aussi de nombreux territoires qui ne sont pas désignés comme aires naturelles protégées, mais qui peuvent offrir une diversité d'activités d'écotourisme: les réserves fauniques, les territoires des pourvoiries à droits exclusifs et ceux des ZEC, les forêts modèles, les forêts habitées, les centres éducatifs forestiers, les parcs régionaux et les terrains sous baux ou vendus par le ministère des Ressources naturelles pour des projets récréotouristiques. Cependant, ces territoires sont généralement soumis à divers types d'exploitation et sont donc très susceptibles aux conflits d'usage (chasse, plein air "motorisé ", foresterie, mines, villégiature) et ne peuvent garantir la constance ni la pérennité de leurs attributs naturels (Couture, 2002 : 43).

Maintenir des secteurs peu accessibles par la route comporte donc certains avantages dont l'atténuation de certains conflits d'usage. De plus, si tous les territoires sont rendus accessibles, les possibilités d'activités futures pourraient être limitées. À l'inverse, il pourrait y avoir des pertes économiques à essuyer par les pourvoiries si leur clientèle s'intéresse plutôt aux territoires peu accessibles. Également, les coûts à long terme liés au contrôle de l'accès ou au braconnage dans les territoires à gestion faunique (réserves fauniques, ZEC, pourvoiries) augmentent avec l'accessibilité au territoire. Tous ces conflits potentiels devraient être envisagés et pourraient être réglés par une meilleure planification qui devra être précédée d'une concertation entre les différents ministères. Force est de reconnaître qu'il y a plus que la matière ligneuse en milieu forestier et que le maintien de régions sauvages est une source de plus en plus importante d'activités économiques. À titre d'exemple, des régions du nord de l'Ontario ont accueilli 153000 touristes, générant 206 millions de dollars en $\mathrm{PIB}$, en retombées directes et indirectes (Ontario Ministry of Economic Development, Trade, and Tourism, 1996 dans Chris Henschel, 2003: 7). Des tels chiffres, qui par ailleurs ne cessent d'augmenter, devraient servir d'incitatif à la conservation de vastes portions représentatives et intactes de forêts ou, du moins, pour un dialogue plus ouvert sur l'aménagement des espaces naturels. En effet, les régions ou les pays qui ont conservé l'intégrité de grands territoires et de milieux naturels ont, dans la plupart des cas, une économie en meilleure santé que les régions qui visent uniquement l'exploitation des ressources (Bourgeois et al., 2006).

Dans les zones où la densité de routes est importante, le deuxième élément de la stratégie proposée est la renaturalisation des routes. L'industrie forestière mentionne qu'une perte des zones productives serait en partie liée au développement du réseau routier (Commission Coulombe, 2004 : 133). En effet, il n'est pas rare de retrouver en forêt boréale exploitée une route à tous les 400 mètres (comme c'est le cas dans plusieurs pays scandinaves). Et si les routes ont une largeur d'une dizaine de mètres (US Army Corps of Engineers, 2004 : 2), soit la largeur totale de la chaussée comprenant aussi les fossés, on compterait pas moins de 3,5\% du territoire dédié strictement au réseau routier. Si le réseau est plus dense ou si les routes sont plus larges (en incluant les zones de débardage, les intersections, les arbres coupés le long des routes, etc.), ce pourcentage peut grimper très rapidement. La biodiversité aussi jouirait des bienfaits d'un accès restreint et de la renaturalisation des routes.

En Ontario, en Alberta et en ColombieBritannique, diverses stratégies sont employées pour refermer les routes afin de limiter les effets néfastes de la circulation (chasse braconnage circulation de véhicules motorisés, fragmentation, etc.) sur la biodiversité. Au Québec, il est encore illégal d'interdire l'accès au territoire, sans compter que plusieurs stratégies d'aménagement forestier comme les coupes partielles et les coupes en mosaïque (Leduc et al., 2002 : 20) requièrent un réseau routier bien développé.

Le dernier élément de la stratégie que nous proposons repose sur des mesures d'atténuation des impacts des routes per- manentes. Certaines mesures sont déjà en vigueur, par exemple laisser des lisières le long des axes d'accès (routes principales et cours d'eau), mais un peu de créativité et une meilleure concertation entre les différents utilisateurs apporteraient de nouvelles solutions. Dans certains secteurs, des «écrans visuels " sont laissés le long des routes secondaires qui servent d'accès aux zones touristiques. Ce type de lisières peut parfois déranger plutôt qu'atténuer les impacts, quand les gens peuvent apercevoir derrière les coupes qui ont été faites. II serait plus judicieux de laisser ces zones ouvertes en variant le degré d'ouverture par des coupes en angles ou des coupes partielles. Une législation moins rigide faciliterait la planification pour satisfaire l'opinion publique plutôt que les administrateurs.

Sheppard et al. (2004) se sont penchés sur des modifications de techniques de coupe totale qui visaient des objectifs de qualité visuelle tout en permettant l'extraction d'un volume élevé de matière ligneuse. Diverses considérations sont mises de l'avant, soit : éviter les patrons réguliers ainsi que des formes géométriques, créer des ouvertures qui ne sont pas toutes visibles d'un seul point d'observation, favoriser les ouvertures étroites, atténuer ou adoucir les lisières de manière à ce qu'il y ait une gradation entre les grands arbres et la zone ouverte et laisser des arbres en rétention (vivants et morts). La rétention d'arbres vivants et morts servirait aussi à assurer le rétablissement rapide de la structure d'une forêt mature et le maintien de plusieurs éléments de biodiversité qui, en soi, peuvent être attrayants pour certains visiteurs. Par ailleurs, on a rapporté que les touristes acceptent plus facilement les coupes lorsque plus d'un quart des arbres restent sur pied après la coupe (British Columbia Ministry of Forests and Range, 2006 : 10).

\section{Planification et inconnus}

Au moment de la planification des routes, il faut déterminer les meilleurs moyens pour assurer la survie à long terme des communautés. Le gouvernement participe à l'aménagement des forêts, assure le développement des communautés et l'économie régionale alors que, traditionnellement, c'était plutôt les industries d'extraction des ressources (forestière, mines, etc.) qui étaient responsables du développement 
des régions et de l'évolution du réseau routier. Or, la situation a changé et les industries, bien qu'elles jouent un rôle majeur, ne sont plus les seuls moteurs de l'économie régionale.

Le rôle des différentes industries dans la survie des communautés est donc remis en question. La planification doit se faire dans une optique de développement durable. L'expansion du réseau routier n'est plus ce qu'elle était il y a trente ans. En effet, l'accessibilité et les effets directs et indirects qui en découlent comportent des avantages et des désavantages, sans compter que les territoires dépourvus de route sont plutôt l'exception que la règle et ce, même en forêt boréale. En conséquence, il semble impératif que plusieurs des éléments de la stratégie énoncée plus haut soient mis de l'avant afin d'assurer le maintien à long terme des différentes options d'utilisation du territoire. On ne peut nier que la foresterie ait été déterminante pour le développement de la forêt boréale, mais l'écotourisme et le tourisme de nature ont joué et continueront de jouer un rôle non négligeable. Pour rester concurrentiel, le Québec doit veiller à ce que les attractions touristiques en forêt soient appuyées par une démarche concertée de planification. La planification des accès routiers ainsi que les effets directs et indirects du réseau routier devront être considérés à plusieurs échelles.

Daniel Kneeshaw est membre du Centre d'Études de la Forêt à l'Université du Québec à Montréal.

Sylvie Gauthier œuvre auprès de Ressources naturelles Canada, Service canadien des forêts, Centre de foresterie des Laurentides.

\section{Notes}

1 Nous aimerions remercier Hélène St-Arnaud pour ses précieux commentaires et informations. La rédaction de ce manuscrit n'aurait pas été possible sans l'aide d'Anh Thu Pham et de Dominic Sénécal. Nous aimerons aussi remercier tous nos collègues qui ont stimulé notre réflexion sur ce sujet et sur d'autres sujets similaires. Nous désirons enfin remercier le Conseil de recherches en sciences naturelles et en génie du Canada (CRSNG) de son appui financier à nos travaux.

2 Zone d'exploitation contrôlée.

\section{Bibliographie}

Bonneau, Josiane, et Daniel D. Kneeshaw (2005), "La représentativité : Un nouveau concept en conservation des ressources forestières ", L'Aubelle no 149, été-automne, p. 29-30.

Bourgeois, Laurence, Daniel D. Kneeshaw, et Gaétane Boisseau (2006), "Les routes forestières au Québec: Les impacts environnementaux, sociaux et économiques ", Vertigo, vol. 6, n², septembre 2006, [http:// www.vertigo.uqam.ca/vol6no2/art16vol6no2/ laurence_bourgeois.html].

British Columbia Ministry of Forests and Range (2006), The Public Response to Harvest Practices in British Columbia at the Landscape and Stand Level, Forest Practices Branch, Victoria, Colombie-Britannique, 47 p., [http:// www.for.gov.bc.ca/hfd/pubs/Docs/Mr/Rec/ Rec038.pdf].

Commission Coulombe (2004), Commission d'étude sur la gestion de la forêt publique québécoise, Rapport, Québec, 314 p., [http://www. mrnf.gouv.qc.ca/publications/forets/consul tation/rapport-coulombe.pdf].

Couture, Maurice (2002), "Nature et tourisme: L'écotourisme au Québec en 2002 ", Téoros, vol. 21, n 3 , automne, p. 43-49.

Fédération des pourvoiries du Québec (2004), Mémoire de la fédération présenté à la commission d'étude sur la gestion des forêts publiques, 17 p., [http://www.fpq.com/medias/ press/memories175.pdf].

Forman, Richard T.T. (2000), « Estimate of the Area Affected Ecologically by the Road System in the United States ", Conservation Biology, vol. 14, n 1, février, p. 31-35.

Henschel, Chris (2003), The Road Less Travelled? A Report on the Effectiveness of Controlling Motorized Access in Remote Areas of Ontario, A Case Study in Temagami, Global Forest Watch Canada Report, 42 p., [http://www. globalforestwatch.org/english/canada/pdf/ Temagami_Audit_Final.pdf].

Leduc, Alain, Christian Messier, Pierre Drapeau, Brian Harvey, Yves Bergeron Louis Imbeau, Alison Munson, Louis Bélanger, Luc Bouthillier, Marcel Darveau, Luc Sirois, et Daniel D. Kneeshaw (2002), "Modification du RNI: une timide ouverture à une gestion plus adaptée aux réalités régionales ", L'Aubelle, n 140, p. 20-21.

Lequin, Marie, et Jean-Bernard Carrière (2003), "Planification de l'accessibilité à l'expérience globale en écotourisme", Téoros, vol.22, n 3, automne, p. 29-36.

Marcoux, Jean-Louis (1993). "Les pourvoiries et l'écotourisme", Franc-vert, vol. 10, n² 2 , mars-avril, p. 18-20.

Mathieu, Daniel (2004), Études sur la valeur économique de l'écotourisme et du tourisme d'aventure, Rapport DBSF, Aventure Écotourisme Québec.
Messier, Christian, et Daniel D. Kneeshaw (1999), "Thinking and Acting Differently for a Sustainable Management of the Boreal Forest», Forestry Chronicle, vol. 75, n 6 , p. 929-938.

Messier, Christian, Brigitte Bigué, et Louis Bernier (2003), "Using Fast-growing Plantations to Promote Forest Ecosystem Protection in Canada», Unasylva, vol. 54, $\mathrm{n}^{\circ}$ 214-215, p. 59-63.

Seymour, Robert S., et Malcom L. Hunter (1992), New Forestry in Eastern Spruce-fir Forests: Principles and Applications to Maine, Maine Agricultural Experiment Station, Orono, Maine, Miscellaneous Publication, MP716, $36 \mathrm{p}$.

Sheppard, Stephen, Paul Picard, et Robert G. D’Eon (2004), «Meeting Visual Quality Objectives with Operational Radial-strip Partial Cutting in Coastal British Columbia: A Postharvest Assessment», Forestry Chronicle, vol. 80, n², mars-avril, p. 215-223.

Société de la faune et des parcs du Québec (2003), Impact économique pour le Québec des dépenses d'exploitation du réseau des gestionnaires de territoires fauniques en 2000 , Rapport Québec, 10 p., [http://www.mrnf. gouv.qc.ca/faune/nature-en-chiffres/pdf/im pact_economique_reseau.pdf].

Tourisme Québec (2003), Écotourisme et tourisme de nature, orientations et plan d'action 2003-2008, Direction du développement des produits touristiques, Gouvernement du Québec, 73 p., [http://www.mdeie.gouv. qc.ca/publications/pdf/etudes/planeco.pdf].

Tourisme Québec (2004), Le tourisme en chiffres 2004, Gouvernement du Québec, 12 p., [http://www.bonjourquebec.com/mto/publi cations/pdf/etudes/tourisme_chiffres2004. pdf].

Trombulak, Stephen C., et Christopher A. Frissell (2000), «Review of Ecological Effects of Roads on Terrestrial and Aquatic Communities", Conservation Biology, vol. 14, n 1, février, p. 18-30.

US Army Corps of Engineers (2004), Provisions for the Construction of Forests Roads in Wetlands in North Carolina, US Department of the Army, Washington, DC, $4 \mathrm{p}$. 